\title{
Prevalence of Veneral Syphilis Among Youth Aged Between 18-35 Years Attending Kisenye Health Centre IV, Kampala District.
}

\author{
Ronald Magezi ${ }^{a}$, Wasswa Amos ${ }^{a}$ \\ a Nsambya Hospital Laboratory school
}

\begin{abstract}
\end{abstract}

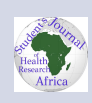

Background:

Syphilis is an infection caused by a spirochete Treponema pallidum transmitted by direct contact with a syphilitic sore on the skin and in mucous membrane. The study was under taken to determine the prevalence of venereal syphilis among youth aged between 18-35years, it also assessed the predisposing factors, prevention and control measures of venereal syphilis.

Methodology:

This was a cross-sectional study conducted among 96 respondents in Kisenyi Health Centre IV between the month of January and February 2020. Simple random sampling method was used to select the 96 respondents whereby the respondents were selected by the first come first serve basis. Questionnaire interview schedule was used for data collection, Venous blood was collected and the Treponema pallidum hem-agglutination assay test kits were used in the diagnosis.

Results:

Out of the 96 respondents who were enrolled in the study 20(20.8\%) were males and 76(76.2\%) were females. Majority of the respondents who were affected were females $04(4.2 \%)$ in the age (18-35). The most predisposing factors to venereal syphilis was Ignorance, the use of drugs, poverty, co-infection, little knowledge about the use of a condom, as well as urban residence were also revealed to be some of the predisposing factors to acquire the disease.

\section{Conclusion:}

The prevalence of venereal syphilis in kisenyi health center IV was found to be higher among the youth especially between the age of $18-25$ years.

Recommendations: ${ }^{a}$

The health care providers should do thorough assessment to address common disease especially cases of venereal syphilis among the youth between 18-35 years.

\footnotetext{
${ }^{a}$ submitted: 3rd/05/2021 accepted: 19/06/2021 email: Skitronie@gmail.com
}

\section{Background of the Study}

Syphilis is an infection caused by a spirochete Treponema pallidum transmitted by direct contact with a syphilitic sore on the skin and in mucous membrane. (Brown and Frank 2003).

A sore can occur in the vagina, anus, rectum, lips and mouth, and it's most likely to spread during oral, anal or vaginal sexual activity. It manifests in three stages that is primary, secondary and tertiary syphilis.

Rarely it can be passed on through kissing and was one of the oldest a well-known disease for which curative treatment is available and efficient.

$60 \%$ of cases affect men who have sex with men or both men and women and were as a result of; 
those who have unprotected sex , men who have sex with men, those with HIV and people with numerous sexual partners

It was also discovered that syphilis was associated with, women with lower education levels, Coinfection with HIV and concurrent sexual partners (mutagoma, 2016).

Men who have sex with men, drug users (use of alcohol, marijuana, cocaine and crack), was also significantly associated with syphilis (Gomes et al., 2017).

Infection with Syphilis is associated with still birth, neonatal death, low immunity, damage to the brain, heart, nerves leading to paralysis, blindness, deafness and death. Globally it was estimated that there are 10 million new syphilis infection among people in the world of which $90 \%$ of the cases occur in limited resource countries (Siraj, 2019)

The reported annual new cases of syphilis in African region were 3.4 million (mutagoma 2012).

In sub-Saharan Africa, 2.9 million people were infected with syphilis, the greatest percentage being women (Hussien, siray \& Birkneh, 2019).

The prevalence of venereal syphilis among the pregnant women in the rural area of Tanzania was $1.6 \%$ and was higher $(2.4 \%)$ among young women aged between 18-35 years (yahya malima et al., 2008).

In Uganda, it was discovered that active syphilis prevalence was $4.3 \%$ between the ages of 18-35 years and this has been observed in places around shores of lake Victoria (Asiki et al., 2011).

In response to the initiative by the ministry of health Uganda in partnership with other nongovernmental organizations much emphasis was put on the control of sexually transmitted Diseases like syphilis through screening of all pregnant youth during antenatal care and providing more testing materials.

Because of this, the researcher found it necessary to undertake a study on the prevalence of venereal syphilis among the youth aged $18-35$ attending Kisenyi Health Centre IV as to address these factors.

A recent study carried out in Uganda showed increased prevalence of syphilis from $1.2 \%$ to $3.7 \%$ (Mutagoma, 2016).

Actions to control and prevent syphilitic infections at different levels of health systems taken for example giving free condoms in most parts of the country, supplying free medicines used in the treat- ment of syphilitic infection and health education (MOH in partnership with Kind Uganda, 2012).

Despite of all the above efforts, re-infection with syphilis, drug resistance to syphilis infection and beliefs among youth who were sexually active that having such a disease makes them known as heroes of the community has created space for syphilis to increase among youth and thus becoming a public health threat.

Because of this, the researcher finds it necessary to undertake a study on the prevalence of venereal syphilis among the youth aged between18-35 attending Kisenyi Health Centre IV as to address these factors.

\section{METHODOLOGY \\ Study Area}

The study was carried out at Kisenyi Health IV which is a public Health Centre in the central region of Uganda under the ministry of health - Uganda. The researcher chose Kisenyi Health Centre IV because it had a history of syphilis victims and also due to the disposing factors in the area of kisenyi. The health Centre is located in the town of Kampala, in Kampala district, approximately 4.0 kilometers by road along Gadhafi road away from Mulago national referral hospital. Kisenyi Health Centre IV is located along Mwanga II Road in Kisenyi in Kampala District. The geographical co-ordinates of kisenyi Health Centre IV are $0.3076^{\circ} \mathrm{N}, 32.5693^{\circ} \mathrm{E}$.

\section{Study Design}

A cross-sectional descriptive study design consisting of quantitative strategies was employed. This was so as to help convert quantitative data into figures which was easy to analyze or as to correlate the theoretical findings with the statistics, and samples were collected from 96 respondents who presented with signs and symptoms of venereal syphilis at kisenyi health Centre IV.

\section{Study Population}

The study was carried out among 96 respondents aged 18-35 who attended kisenye Health center IV with the signs and symptoms of venereal syphilis during the study period in Kampala district.This was because they had a history of syphilis victims

\section{Inclusion Criteria}

The study involved all patients between the ages 18-35 years with signs and symptoms of venereal syphilis who attended kisenyi Health Centre IV during the study period, those who were residents of 
that district and those who consented to take part in the study

\section{Exclusion Criteria}

The study excluded all patients below 18 and above 35 years with no signs and symptoms of Syphilis and those who did not sign the consent form.

\section{Sample Size Determination}

The study was carried on 96 respondents. The sample size was defined using the

Kish Leslie formula in the year 1964.

$\mathrm{n}=\mathrm{Z} 2 \mathrm{pq}$

E2

Where $\mathrm{N}=$ sample size to be interviewed.

$\mathrm{Z}=$ standard normal deviation corresponding to

95\% confidence which was equal to 1.96

$\mathrm{P}=$ Prevalence of syphilis, take it to be $50 \%$ since it is not known.

$d=$ accepted sampling error. Which will be 0.1

$\mathrm{Q}=1-\mathrm{p}=1-0.5=0.5$

Therefore: $n=1.96^{2} \times 0.5(1-0.5)$

$0.1^{2}$

$\mathrm{N}=3.8416 \times 0.5 \times 0.5$

0.01

$\mathrm{N}=3.8416 \times 0.25$

0.01

$=96$

Therefore, $\mathrm{N}=96$ respondents

\section{Sampling technique}

Simple random sampling method was used to select the 96 respondents where by the respondents were selected by the first come first serve basis until 96 respondents were got. This facilitated selection of the subjects without bias from the study population.

\section{Sampling procedure}

This was done by use of papers having numbers where respondents willing to participate were allowed to pick randomly as to obtain the required number of respondents. Many small papers about 150 papers were provided having numbers written on them, folded and put in a box and the box shaken. Respondents were allowed to pick one paper each randomly and those from number 1 to 96 were considered

\section{Data collection method}

Questionnaire interview schedule was used for data collection since the population area had both literate and illiterate individuals, this was in a way that the researcher and the research assistants asked the illiterate individuals questions and then wrote answers as stated by the respondents. For the case of literate individuals, they were given questionnaires to answer for themselves which were collected and cross-checked if all the questions were answered to avoid incomplete questionnaires. Those that were incompletely filled were to be returned to the respondents to fully fill them before they left the health Centre.

\section{Data collection tool}

A pretested questionnaire was used as a Data collection tool to collect socio-demographic data ,factors associated with the prevalence of venereal syphilis among the youth and prevention and control of venereal syphilis.

\section{Data collection procedure}

With the help of research assistants, the questionnaires were issued to the respondents to fill in appropriately and the illiterate respondents were helped to fill in the questionnaires.

Data concerning the test results was captured from the laboratory request form when the testing was done.

\section{Laboratory diagnosis}

Venous blood was drawn from the respondent by the researcher into a well labelled red top bottle with the respondent's number. Then centrifuged and the serum was obtained and used for testing. The duration for the test was to depend on the manufacturer of the test kits being used.

Treponema pallidum hem-agglutination assay test kits were used in the diagnosis. And the results were reported as positive or negative.

\section{Piloting the study}

The pilot of study was to assess the viability of data collection instruments and procedures, as well as sampling procedures

\section{Quality control}

The questionnaire were pretested in a nearby health center in Kampala district with the same settings as kisenyi health Centre IV which enabled me to check and quality control the questionnaire for any mistakes, understanding and then improve them accordingly.

All the boxes for the test kits to be used were checked for their performance, expiry date and if necessary for their packaging.

All positive samples were retested for confirmation by some senior laboratory personnel at Kisenyi health Centre IV.

The research assistants was trained first before helping out the respondents in the research study. 


\section{Data analysis and presentation}

Data obtained from the study was analyzed using computer Microsoft word, Microsoft excel and presented in a descriptive form of pie chart, tables and graphs.

Respondents were recorded and issued laboratory numbers and later the results filled in the laboratory result book.

\section{Data management}

All the information collected from the study was kept in a lockable cupboard and access only granted to the researcher.

The questionnaires were also rechecked after answering to avoid incomplete forms before the respondents leave the health center.

\section{Ethical consideration}

An introductory letter was given to me the researcher by the research and ethics committee of Nsambya Hospital training school after approval of my research proposal which was taken to relevant authorities like the dirctor of public health and enviromental service KCCA in charge for approval and permission to carry out research in that area at that hospital. Pre-exercise sensitization was done to enable patients understand the purpose of the research so as to enable them make right decision. Respondents were requested to consent either by signature or thumb print or verbals.

Confidentiality of the respondents was clearly observed.

To ensure confidentiality, laboratory numbers were used but not their names and the results were disclosed to the researcher, respondent and medical practitioner for appropriate treatment for the youth tested positive.

This was accompanied by a school identity card that confirmed that the researcher was really a student in Nsambya hospital training school.

\section{Study limitation}

The researcher was faced with a problem of finances especially for typing, printing, photocopying and transport during the period of data collection.

Respondents asking to be paid before participation in the study, limited time, bias, misconception

Getting current literature of Uganda's context which was not readily available

Language barrier as some respondents were not been literate

\section{Dissemination of results}

Research reports were distributed to the research and ethics committee of Nsambya hospital training school, a copy to Uganda Allied Health Examination Board, a copy to my supervisor, a copy to my Sponsors/ benefactors and a copy of my personal file.

\section{DATA ANALYSIS AND PRESENTATION}

\section{Source: primary data, February 2020}

From table 4.1.2, it is seen that in terms of age, $60(62.5 \%)$ respondents revealed were between the age of $18-25$ years, and 04 (4.2\%) were syphilis positive and 56 (58.3\%) being syphilis negative, 16 (16.7\%) respondents revealed were between the age of 26-30 years, where 01 (1.0\%) were positive for syphilis and $15(15.6 \%)$ were negative whereas the remaining $20(20.8 \%)$ respondents revealed were between $31-35$ years, where all of them tested syphilis negative (20.8\%).

In terms of gender, most of the respondents76 (79.2\%) respondents were females where 03 (3.1\%) of them tested syphilis positive, 73 (76.0\%) tested syphilis negative and the remaining 20 (20.8\%) respondents were males where $02(2.1 \%)$ tested syphilis positive and the remaining 18 (18.8\%) tested syphilis negative.

For tribe, 60 (62.5\%) respondents revealed that they were Baganda, where $01(1.0 \%)$ tested syphilis positive and 59 (61.5\%) tested negative, 20 (20.8\%) respondents revealed they were Basoga, where only 01 (1.0\%) tested positive for syphilis and 19 $(19.8 \%)$ tested negative, 14 (14.6\%) respondents revealed were Banyankole where by 03 (3.1\%) tested syphilis positive whereas $11(11.5 \%)$ were negative and only $02(2.1 \%)$ respondents revealed were of other tribes where they all tested syphilis negative (2.1\%).

In case of religion, 45 (46.8\%) respondents revealed that they were Catholics, whereby they all tested syphilis negative (46.8\%), 30 (31.3\%) respondents answered that they were Anglicans, and they all tested syphilis negative (31.3\%), 15(15.6\%) revealed that they were Muslims where 03 (3.1\%) tested syphilis positive and the remaining 12 (12.5\%) being negative and 06 (6.3\%) respondents answered that they were of other tribes they did not specify where by $02(2.1 \%)$ tested syphilis positive and the remaining syphilis negative 4 (4.2\%). 
Table 1. 2 showing social- demographic factors of respondents

\begin{tabular}{lllll}
\hline $\begin{array}{l}\text { Vari- } \\
\text { able }\end{array}$ & Category & Frequency $(n=96) \%$ & \% syphilis positive & \% syphilis negative \\
Age & $18-25$ 26-30 31-35 & $\begin{array}{l}60(62.5 \%) 16(16.7 \%) \\
20(20.8 \%)\end{array}$ & $\begin{array}{l}04(4.2 \%) 01(1.0 \%) \\
(00 \%)\end{array}$ & $\left.\begin{array}{l}56(58.3 \%) \\
20(20.8 \%)\end{array}\right)$ \\
Gender & Male Female & $20(20.8 \%) 76(79.2)$ & $02(2.1 \%) 03(3.1 \%)$ & $18(18.8 \%) 73(76.0 \%)$ \\
Tribe & Baganda Basoga & $20(20.8 \%) 14(14.6 \%)$ & $01(1.0 \%) 03(3.1 \%)$ & $19(19.8 \%) 11(11.5 \%)$ \\
& Banyankole Others & $60(62.5 \%) 02(2.1 \%)$ & $01(1.0 \%) 00(00 \%)$ & $59(61.5 \%) 02(2.1 \%)$ \\
Reli- & Catholic Anglican & $45(46.8 \%) 30(31.3 \%)$ & $00(00 \%) 00(00 \%) 03$ & $45(46.8 \%) 30(31.3 \%)$ \\
gion & Muslims Others & $15(15.6 \%) 06(6.3 \%)$ & $(3.1 \%) 02(2.1 \%)$ & $12(12.5 \%) 04(4.2 \%)$ \\
Marital & Single Married & $54(56.3 \%) 32(33.3 \%)$ & $04(4.2 \%) 00(00 \%) 01$ & $50(52.1 \%) 32(33.3 \%)$ \\
status & Divorced & $10(10.4)$ & $(1.0 \%)$ & $09(9.4 \%)$ \\
\hline
\end{tabular}

While in terms of marital status, 54 (56.3\%) respondents revealed that they were single, where by $04(4.4 \%)$ tested syphilis positive and the remaining 50 (52.1\%) tested negative for syphilis, 32 (33.3\%) respondents revealed that they were married all tested syphilis negative, and only 10 (10.4\%) respondents revealed that they were divorced 01 $(1.0 \%)$ tested syphilis positive and the remaining 09 (9.4\%) tested negative.

\section{Prevalence of venereal syphilis}

\section{Source: primary, February 2019}

From the figure 1 above, shows that the prevalence of venereal syphilis in Kisenyi Health Centre IV was about $5.0 \%$ and those without venereal syphilis 95\%.

\section{Factors associated with venereal syphilis Source: Primary data, February 2020}

From the table 4.2 .1 shows that $10(10.4 \%)$ respondents revealed that they had never been tested before and when they were tested, 02 (2.1\%) respondents tested syphilis positive and the remaining $08(8.3 \%)$ respondents tested syphilis negative and those of the 86 (89.6\%) had never been tested before, when they were tested, 03 (3.1\%) respondents tested syphilis positive and the remaining $83(86.5 \%)$ tested syphilis negative.

Of the $80(83.3 \%)$ respondents who revealed that they were from the urban area, 05 (5.2\%) tested syphilis positive and the remaining 75 (78.1\%) tested syphilis negative whereas the remaining 16 (16.7\%) respondents revealed that they were living rural when they were tested, they all tested syphilis negative (16.7\%).

Concerning the use of condoms, of the 06 (6.3\%) respondents who revealed that they always use condoms, when they were tested, 05 (5.2\%) re- spondents tested syphilis positive and 01 (1.0\%) tested syphilis negative but of the $90(93,7 \%)$ respondents who revealed that they do not use condoms, when tested, all the 90 (93.8\%) respondents tested syphilis negative.

06 (6.3\%) respondents who revealed had a co infection, 05 (5.2\%) tested syphilis positive 01 (1.0\%) tested syphilis negative while the 91 (94.8\%) respondents who revealed that they had no any other STI, they all tested syphilis negative.

Of the 04 (4.2\%) respondents who revealed that they use drugs of abuse, all tested syphilis positive and the remaining while of the 92 (95.8\%) respondents who revealed that they do not use any drug of abuse, when tested, 01 (1.0\%) respondents tested syphilis positive and the remaining 91 (94.8\%) tested syphilis negative.

Of the 56 (58.3\%) respondents who revealed they had more than one sexual partner, when they were tested they all tested syphilis negative and of the $40(41.7 \%)$ respondents who revealed had more than one sexual partner, when they were tested, $05(5.2 \%)$ respondents tested syphilis positive and the remaining 35 (36.5\%) tested syphilis negative.

\section{PREVENTION AND CONTROL OF VENEREAL SYPHILIS}

\section{Source: primary data February 2020}

Concerning the use of condoms, of the $06(6.3 \%)$ respondents who revealed that they always use condoms, when they were tested ,05(5.2\%) respondents tested syphilis positive and 01 (1.0\%) tested syphilis negative but the $90(93.7 \%)$ respondents who revealed that they do not use condoms, when tested all the $90(93.8 \%)$ respondents tested syphilis negative. 


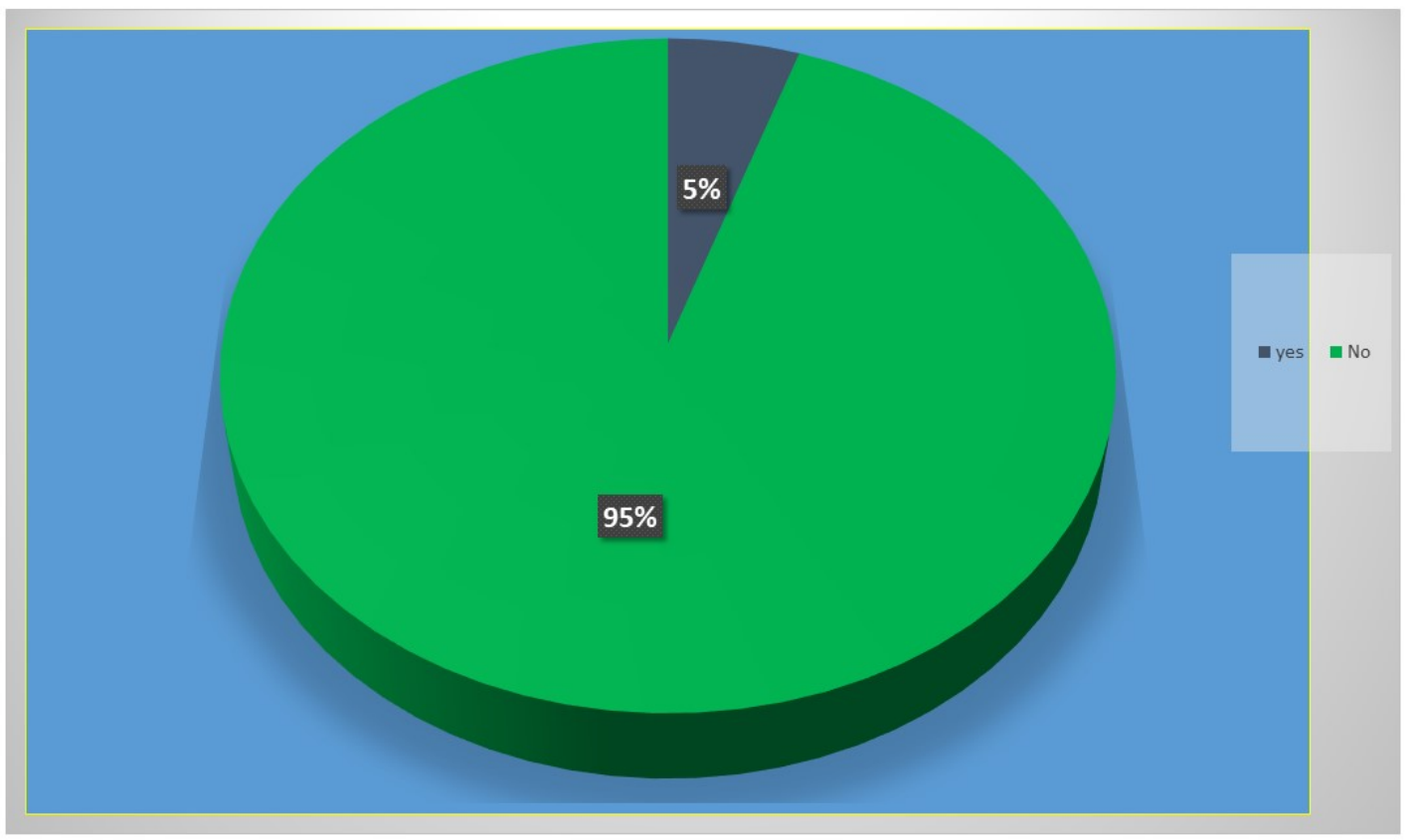

Figure 1. a pie chart showing prevalence of venereal syphilis.

\section{Table 2. howing factors associated with venereal syphilis}

\begin{tabular}{|c|c|c|c|c|}
\hline Variable & Category & $\begin{array}{l}\text { Frequency } \\
(n=96) \%\end{array}$ & $\begin{array}{l}\text { \% syphilis } \\
\text { positive }\end{array}$ & $\begin{array}{l}\text { \% syphilis } \\
\text { negative }\end{array}$ \\
\hline $\begin{array}{l}\text { Have you ever gone to be tested } \\
\text { for syphilis? }\end{array}$ & Yes No & $\begin{array}{l}10(10.4 \%) 86 \\
(89.6 \%)\end{array}$ & $\begin{array}{l}02(2.1 \%) 03 \\
(3.1 \%)\end{array}$ & $\begin{array}{l}08(8.3 \%) 83 \\
(86.5 \%)\end{array}$ \\
\hline Where do you stay? & Urban Rural & $\begin{array}{l}80(83.3 \%) 16 \\
(16.7 \%)\end{array}$ & $\begin{array}{l}05(5.2 \%) 00 \\
(00 \%)\end{array}$ & $\begin{array}{l}75(78.1 \%) 16 \\
(16.7 \%)\end{array}$ \\
\hline Do you always use condoms & Yes No & $\begin{array}{l}06(6.3 \%) 90 \\
(93.7 \%)\end{array}$ & $\begin{array}{l}05(5.2 \%) 00 \\
(00 \%)\end{array}$ & $\begin{array}{l}01(1.0 \%) 90 \\
(93.8 \%)\end{array}$ \\
\hline Do you have any other STI & Yes No & $\begin{array}{l}06(6.3 \%) 91 \\
(94.7 \%)\end{array}$ & $\begin{array}{l}05(5.2 \%) 00 \\
(00 \%)\end{array}$ & $\begin{array}{l}00(1.0 \%) 91 \\
(94.8 \%)\end{array}$ \\
\hline Do you use any drug of abuse? & $\begin{array}{l}\text { Use drugs Do not } \\
\text { use drugs }\end{array}$ & $\begin{array}{l}04(4.2 \%) 92 \\
(95.8 \%)\end{array}$ & $\begin{array}{l}04(4.2 \%) 01 \\
(1.0 \%)\end{array}$ & $\begin{array}{l}00(00 \%) \\
91(94.8 \%)\end{array}$ \\
\hline $\begin{array}{l}\text { How many sexual partners do } \\
\text { you have? }\end{array}$ & One More than one & $\begin{array}{l}56(58.3 \%) 40 \\
(41.7 \%)\end{array}$ & $\begin{array}{l}00(00 \%) 05 \\
((5.2 \%)\end{array}$ & $\begin{array}{l}56(58.3 \%) 35 \\
(36.5 \%)\end{array}$ \\
\hline
\end{tabular}

Table 3. showing the prevention and control of venereal syphilis

Variable Category Frequency $(\mathrm{n}=96) \% \quad \%$ syphilis positive $\%$ syphilis negative

Table 4. showing the prevention and control of venereal syphilis

\begin{tabular}{lllll}
\hline Do you always use condoms & Yes No & $06(6.3 \%)$ & $90(93.7 \%)$ & $05(5.2 \%)$ \\
\hline
\end{tabular}




\section{DISCUSSION, CONCLUSION AND RECOMMENDATION}

\section{Discussion}

Syphilis is a chronic infectious disease caused by the spirochete Pallidum. It has Signiant long term morbidity for the youth, and can cause server complications in pregnancy, which may result in spontaneous abortion, still birth and other outcomes including congenital syphilis, congenital syphilis results in serious sequelae in live born infected children (Genc\&ledger, 2000)

\section{Prevalence of venereal syphilis}

The prevalance of venereal syphilis was found to be higher among females 03(3.1\%) than in males 02(2.1\%) in Kisenyi Kampala compared to the earlier studies that was done in Uganda especially around the shores of Lake Victoria in which the prevalance was found to increase from $1.2 \%$ in youth to 3.7\% (Mutagoma, 2016).

The prevalence was found to be more among the youth between the age of 18-25 years who were 04(4.2\%) compared to those between the age of 31-35years who were $00(00 \%)$ and more especially among the, uneducated, the poor and those living in urban areas, those under the influence of drugs, those with co infection. This was attributed to the youth in this age group being sexually active.

The prevalence was also found to be more among the single/unmarried04(4.2\%) as compared to the married $00(00 \%)$ as they may be having multiple sexual partners which pre disposes them more to the risk of infection.

The prevalence was also found to be more prevalent among the Muslims who were 03(3.1\%)compared to other religion who were $02(2.1 \%)$ since they take it being circumcised can protect them from acquiring infection and hence didn't mind about safe sex practices.

The prevalence was also found to be more prevalent among the Basoga who were 03(3.0\%) compared to other tribes who were $00(00 \%)$.

\section{Social- demographic factors}

The study approached 96 respondents that attended kisenyi Health Centre IV during the study period

Age between $18-25$ having a frequency60(62.5\%) of which $04(4.2 \%)$ respondents tested syphilis positive and 56(58.3\%)respondents tested syphilis neg- ative were found to be associated with venereal syphilis since this age is more sexually active.

Marital status is another factor that was also found to be associated with venereal syphilis since single respondents when tested were more with the disease since they are associated with having multiple sexual partners. This finding is in line with the study that was done by other researchers (Gomes, et al., 2017)

\section{Factors associated with venereal syphilis.}

In terms of marital status, it was found out that respondents who were singe, were at a higher risk of acquiring the disease since they have multiple sexual partners especially the youth and this is why most of the victims were between 18-25years compared to those were married and could be having only one sexual partner for instance of the 56 respondents who revealed that they had more than one sexual partner, when they were tested, 02 respondents tested positive for syphilis and this can be compared with the earlier studies that were carried out by other researchers (Gomes, et al., 2017)

Among other factors that were revealed by respondents also to be associated with venereal syphilis were: education was also found to be associated with the disease since it was also found to be more prevalent among the uneducated compared to the educated individual. this is because educated people are more literate and can easily think about protective measures as well as their high bargaining power for safe sex. This is in comparison with the earlier studies of self-efficacy of more educated women in negotiating safe sex and possibly having other source of income other than sex work (Mutagoma, 2016)

Some respondents also revealed that poverty could force them to look for alternative source of income and this could also expose them to such sex activities hence at risk of getting the infections

Residence was revealed to be associated with syphilis in which it was found out that those individuals in urban areas of $80(83.3 \%), 05(5.2 \%)$ respondents had the disease compared to those who were from rural areas which is about00 (0.0\%) higher in urban areas more among young women than men. This was attributed to having multiple sexual partners, this finding is in line with the study that was done by researchers (gomes, et al., 2017)

Concerning the use of drugs, it was also found to be another associated risk factor, of the $04(4.2 \%)$ respondents who revealed that they had ever used 
drugs of abuse, all tested syphilis positive and the remaining $01(0.1 \%)$ who revealed that didn't use drugs of abuse tested syphilis positive. The use of drugs exposes someone to the risk of getting the disease and this can be seen in comparison with earlier studies where it was found out that the toxicity effect of the drugs alters the adjustment which leads to engagement in unsafe sex (Mutagoma M,2016). Alcohol use as an example is associated with high rate of unprotected intercourse, failure to use condoms appropriately and increased frequency of sexual activity.

Con-infection was revealed by some respondents also to be another factor since con-infection weakens one's body immune system hence increasing chances as one can easily get the disease if someone gets unsafe sex with an infected person and this is in accordance with the study that was carried out by the earlier researches (Asiki G, et al., 2011) in which of the two respondents who revealed were con-infected with HIV, when tested also had syphilis

\section{Conclusion}

The major conclusion that can be derived from this study are:

There was a high prevalance rate of $4.2 \%$ among the youth aged $18-25$ years. Ignorance especially among those with low education level, marital status of being single/unmarried, the use of drugs, poverty, little knowledge about the use of condoms as well as urban residence were the predisposing factors. These factors must be considered for proper implementation of any prevention and control strategies regarding venereal syphilis among the youth aged 18-35 attending Kisenyi Health Centre IV

\section{Recommendation}

Basing on the study findings, the following recommendations should be considered.

To the youth attending Kisenyi Health centre IV

They should ensure that they go for screening on every hospital visit.

In case they are found positive for venereal syphilis, they should adhere to treatment provided by the health care personnel

To the administration of Kisenyi Health Centre IV

The health care administration should instruct its health care team to sensitize all the youth on every hospital visit on predisposing factors, control and preventive measures of venereal syphilis to lower the prevalance.

The health care team at the health centre should inform all the youth on the effects of venereal syphilis in order to increase awareness hence help eradicating

Health education should be emphasized among the youth through reproductive health talk on media, at health facilities to create awareness of the dangers that may result, encourage them to always have safe sex and provide materials like condoms in public accessible places as well as improve on health facilities especially in urban areas.

\section{To the government and the ministry of} health

The government should increase their budget for the health department so that a wider range of venereal syphilis causing agents can be diagnosed and confirmed by the laboratory methods

The $\mathrm{MOH}$ should support the youth with income generating projects to especially women to increase their economic strength hence preventing them from un healthy behaviors like prostution which expose them to the infection.

There is need to carry out a wider and more detailed research to establish more information about venereal syphilis to aid in the diagnosis

The $\mathrm{MOH}$ should organize programs to train health care professionals on predisposing factors, prevention, control measures and effects of venereal syphilis in order to enable them pass on the information to their clients at different health centers

\section{ACKNOWLEDGEMENT}

Thank Almighty God for enabling me to pursue my studies throughout

I further express gratitude to the school administration of Nsambya Hospital Laboratory school for academic atmosphere offered during my period of study at the institute. In the same context, am thankful to the supervisor of my research for the endless guidance from the initials stages of formulating the topic to the final stages of report submission.

I equally register my appreciation to the administration of Kisenyi Health Centre IV for having accepted and helped me endlessly during my data collection at the Hospital Laboratory department. 
Am greatly indebted to my family for enduring countless period of my absence while I sought better and promising horizons for better happiness.

Special thanks to my friends and schoolmates especially the class of DMLT 2017-2020 for the unending discussion, revisions and deliberations as we sought to discover the unknown

\section{ABBREVIATION AND ACRONYMS OPERATIONAL DEFINITIONS}

Aggregated: Added together as a total or single amount.

Averted: To prevent something bad or dangerous from happening.

Health: A state of physical, mental and social well-being of an individual. It can also be a state in which all the body systems of an individual are in proper functioning.

Immunological response: A bodily defense reaction that recognizes an invading substance and produces antibodies against it.

Laboratory: A special room where all scientific tests are carried out.

Morbidity: The state of being ill.

Mortality: Number of deaths in a particular situation or period of time

Prevalence: Is the number of people (proportion) of a population who have a specific characteristic in a given period of time.

Population: Is the number of people living in an area at a particular time.

Plummeted: To fall suddenly and quickly from a high level or position.

Probable active syphilis: seroreactivity in both non-Treponema and Treponema tests.

Risk: A likelihood of occurrence of a potential hazard.

Sexually Transmitted Diseases: Are diseases that are transmitted from an infected person to another through sexual intercourse for example HIV, Syphilis.

Spirochete: A genus of bacterium that cause Syphilis

Treponema pallidum: A small organism (bacterium) that causes syphilis.

\section{References:}

1). Asiki, G., Mpendo, J., Abaasa, A., Agaba, C., Nanvubya, A., Nielsen, L., Seeley, J., Kaleebu, P., Grosskurth, H., \& Kamali, A. (2011). HIV and syphilis prevalence and associated risk factors among fishing communities of Lake Victoria, Uganda. Sexually transmitted infections, 87(6), 511-515. https://doi.o rg/10.1136/sti.2010.046805

2). Brown, D. L., \& Frank, J. E. (2003). Diagnosis and management of syphilis. American family physician, 68(2), 283-290 Siraj Hussen, Birkneh Tilahun Tadesse, "Prevalence of Syphilis among Pregnant Women in Sub-Saharan Africa: A Systematic Review and Meta-Analysis", BioMed Research International, vol. 2019, Article ID 4562385, 10 pages, 2019.

3). Genç, M., \& Ledger, W. J. (2000). Syphilis in pregnancy. Sexually transmitted infections, 76(2), 73-79.

4). Gomez, G.B., Kamba, M.L., Newman L.M., Mark, J., Bountent, N., et al. (2013) untreated maternal syphilis and adverse outcomes of pregnancy: a systematic review and meta-analysis. Bull world health orga91. 217-226

5). PMid:31392211 PMCid:PMC6662498 YahyaMalima, K.I., Evjen-Olsen, B., Matee, M.I. et al. HIV1, HSV-2 and syphilis among pregnant women in a rural area of Tanzania: Prevalence and risk factors. BMC Infect Dis 8, 75 (2008). https://doi. org/10.1186/1471-2334-8-75 PMid:18513451 PMCid:PMC2423369

6). World Health Organization ;( 2015), Global incidence and prevalence of selected sexually transmitted infections- Geneva, Switzerland Mwumvaneza Mutagoma, Eric Remera, Dieudonné Sebuhoro, Steve Kanters, David J. Riedel, Sabin Nsanzimana, "The Prevalence of Syphilis Infection and Its Associated Factors in the General Population of Rwanda: A National Household-Based Survey", Journal of Sexually Transmitted Diseases, vol. 2016, Article ID 4980417, 8 pages, 2016. 
Table 5. ABBREVIATION AND ACRONYMS

\section{CDC : Center for Disease Control}

CS : Congenital Syphilis

MSM \% : : Men who have sex with men Percentage

STIS : Sexually Transmitted Infections

SSA : Sub-Saharan Africa

UAHEB : Uganda Allied Health Examination Board

WHO : World Health Organization 